Ophthalmic Physiol Opt. 2015 September ; 35(5): 530-539. doi:10.1111/opo.12232.

\title{
Hazard detection with a monocular bioptic telescope
}

\author{
Amy L. Doherty, Eli Peli, and Gang Luo \\ Schepens Eye Research Institute, Massachusetts Eye and Ear, Harvard Medical School, Boston, \\ USA
}

\begin{abstract}
Purpose-The safety of bioptic telescopes for driving remains controversial. The ring scotoma, an area to the telescope eye due to the telescope magnification, has been the main cause of concern. This study evaluates whether bioptic users can use the fellow eye to detect in hazards driving videos that fall in the ring scotoma area.
\end{abstract}

\begin{abstract}
Methods-Twelve visually impaired bioptic users watched a series of driving hazard perception training videos and responded as soon as they detected a hazard while reading aloud letters presented on the screen. The letters were placed such that when reading them through the telescope the hazard fell in the ring scotoma area. Four conditions were tested: no bioptic and no reading, reading without bioptic, reading with a bioptic that did not occlude the fellow eye (nonoccluding bioptic), and reading with a bioptic that partially-occluded the fellow eye. Eight normally sighted subjects performed the same task with the partially occluding bioptic detecting lateral hazards (blocked by the device scotoma) and vertical hazards (outside the scotoma) to further determine the cause-and-effect relationship between hazard detection and the fellow eye.

Results-There were significant differences in performance between conditions: $83 \%$ of hazards were detected with no reading task, dropping to $67 \%$ in the reading task with no bioptic, to $50 \%$ while reading with the non-occluding bioptic, and $34 \%$ while reading with the partially occluding bioptic. For normally sighted, detection of vertical hazards (53\%) was significantly higher than lateral hazards (38\%) with the partially occluding bioptic.
\end{abstract}

Conclusions-Detection of driving hazards is impaired by the addition of a secondary reading like task. Detection is further impaired when reading through a monocular telescope. The effect of the partially-occluding bioptic supports the role of the non-occluded fellow eye in compensating for the ring scotoma.

\section{Keywords}

bioptic driving; bioptic telescope; hazard perception; ring scotoma

Correspondence: Gang Luo gang_luo@meei.harvard.edu.

Disclosure

A Doherty and G Luo report no conflicts of interest and have no proprietary interest in any of the materials mentioned in this article. $\mathrm{E}$ Peli has rights in a patent for a bioptic telescope design. 


\section{Introduction}

Small spectacle-mounted telescopes, known as bioptics, enable people with reduced visual acuity to see details of distant objects and can be used as driving aids. There is some evidence for their safe use for driving, ${ }^{1,2}$ and also reports to the contrary. ${ }^{3,4}$ Despite their controversial use, bioptic telescopes are permitted for driving in 43 states in the US, ${ }^{5}$ the Netherlands, ${ }^{6,7}$ and Quebec, Canada. The number of jurisdictions permitting them has increased substantially over the last decade. The wearer spends most of the time viewing through the unmagnified carrier lens, and when detailed information is needed, makes a brief head tilt to view through the telescope. ${ }^{8}$ While viewing through a telescope, the magnified field-of-view on the retina covers part of the image otherwise available in the unmagnified view creating a ring scotoma, an annulus-shaped area of the field that is not visible to the telescope eye. Some claim that the ring scotoma impairs detection of important objects and events, ${ }^{9,10}$ while others argue that with a monocular telescope, the fellow (nontelescope) eye can compensate. ${ }^{1,11}$ In conventional perimetry (bright spot on dimmer white background), the fellow eye can detect stimuli presented in the ring scotoma area when viewing binocularly. ${ }^{4,12,13}$ In more complex environments such as driving the difference in retinal images due to the magnification difference between the eyes may cause binocular rivalry or suppression, and therefore may prevent the fellow eye from compensating. ${ }^{14}$ Our previous studies showed that, when viewing through a monocular bioptic telescope, the detection performance of the fellow eye for stimuli in the ring scotoma area on patterned backgrounds is not affected by the telescope. ${ }^{15,16}$ While our previous studies represented more complex visual conditions than conventional perimetry, including the potential for rivalry and suppression, the images were static with stimulus onset the only temporal change. Stimulus onset is well known to capture attention in rivalrous conditions. ${ }^{17}$ The current study addresses some limitations of our prior studies by evaluating detection of realistic hazards in real world driving videos, in which the stimuli (driving hazards) gradually develop rather than suddenly appear (as in the perimetry-like stimuli in prior studies). It also requires recognition of the hazardous nature of the situation rather than a mere detection of light increment. This study is therefore more relevant to the driving environment in which bioptic telescopes are used.

Hazard perception tests evaluating the ability to anticipate, detect, and respond appropriately to driving relevant events, ${ }^{18-20}$ are required for licensure in the UK and some states in Australia. ${ }^{18}$ Hazard perception is usually evaluated by having individuals identify hazards in video clips recorded from a dashboard camera in an urban setting. In this study, we used hazard perception training videos to evaluate the ability of bioptic users to detect hazards, with and without bioptic telescopes, while performing a task similar to road sign reading, one of the main tasks bioptics are used for. ${ }^{8}$ These training videos are used by licensure candidates to prepare for the official test. We hypothesised that while realistic hazard detection might be affected by the ring scotoma, due to increased predominance of the attended telescope eye, the fellow eye could still compensate for the ring scotoma. To prove the cause-and-effect relationship between hazard detection and the fellow eye, a monocular bioptic that partially occluded the fellow eye was used to determine if the detection of hazards could be manipulated by controlling the occlusion in the fellow eye. 


\section{Methods}

\section{Experiment procedure}

Visually impaired participants performed four conditions of hazard detection: no bioptic and no reading task (as used in the administration of the test in the UK and Australia), letter reading task without a bioptic, reading task through the non-occluding bioptic design, and reading task through the partially occluding bioptic design. In order for hazards to remain in the ring scotoma while they evolve, participants were required to keep viewing through the telescope. Thus, a reading-like task longer than might be commonly expected when reading a road sign was designed. The reading task without a bioptic serves as a control condition to measure the impact of attention demanded by the reading task alone. It also represents attentional demands similar to reading a text message on a head-up or smart glasses display while driving. Participants watched each video only once. The pairing of video set and test condition and the order of test conditions was counterbalanced across participants. To compare performance with the UK test, a group of normally sighted subjects completed all four video sets in the no reading task condition and performance was averaged across video sets.

In each condition, participants pressed a button as soon as they detected a hazard. Before testing, participants were given a short practice session to familiarise them with the concept of hazards in these videos. They sat 1.5 metres from a large rear-projection screen so the field of view $\left(58 \times 45^{\circ}\right)$ was similar to the field of view depicted in the video.

\section{Reading task}

Figure 1 illustrates the reading task. Single black alpha-numeric characters on a white square background with a red border, appeared at random times and locations superimposed on each video. The characters were high contrast to be easily seen and located through the telescope. Throughout the 18 videos in each set, 30 sequences of characters were presented (one or two times in each video) with 50\% of the character sequences coinciding with a hazard. When the sequence occurred with a hazard, its duration equalled the hazard duration. For sequences without hazards, the duration was within the range of the average hazard duration plus or minus the standard deviation (5.7 $\pm 1.7 \mathrm{~s}$ ). Sequences could appear at any time during the video with the constraints that if occurring with a hazard, they occurred at the time of the hazard; otherwise they began at least $5 \mathrm{~s}$ after the start of the video, finished at least $5 \mathrm{~s}$ before the end of the video and had a minimum of $5 \mathrm{~s}$ between sequences. Characters were positioned in the scene at least $5^{\circ}$ from the edge of the projected scene and $15^{\circ}$ from the centre of the area covered from the start to end of the hazard (e.g. all frames in Figure 1c), in one of eight cardinal directions chosen randomly, yet balanced between conditions (Figure 1a). This position caused the hazard to fall in the ring scotoma area when the telescope was centred on the character box (Figure 1b). When not coinciding with a hazard, characters were positioned at random locations within the range of hazard locations so it was not obvious which corresponded with hazards. The timing and location randomisation were determined using custom MATLAB programs. 
In each reading task, participants called out single letters embedded among a series of numbers. Characters changed every $320 \mathrm{~ms}$ (Figure 1c) and an audible beep occurred $1 \mathrm{~s}$ before the first character to alert participants. Participants were instructed to call out all the letters they saw and were informed that any number of letters could appear at any time during the sequence. Character sizes used could be comfortably read by participants (Table 1). For all but three participants the magnification with the partially occluding bioptic was higher, thus the reading-like task was easier with this telescope, making that condition slightly less demanding.

\section{Hazard detection Videos}

The videos were selected from a commercial DVD used to prepare drivers for the UK hazard perception licensing test (Driving Test Success Hazard Perception: Imagitech Ltd, Swansea, UK). Videos were mirror reflected using Adobe Premiere so that the cars appear to drive on the right side of the road. Only daytime videos with pedestrian or vehicle hazards were selected, to make it easier for participants to understand what hazards could appear. A total of 72 videos (out of 400) were used, divided into four sets of 18 videos, for the four test conditions so that participants saw each video once. Video sets were matched to the best of our ability for road type and hazard type, size, duration, location and time in the scene. Each video was approximately $1 \mathrm{~min}$ in length. Fifteen of the 18 videos in each set contained hazards, three contained no hazards.

\section{Hazards}

Hazards included in this study were pedestrians or vehicles that would cause the driver to change speed, course, or direction. They included pedestrians crossing the road, cars turning into the drivers' lane, or merging with insufficient gap. Hazards could appear anywhere in the scene at any time throughout a video. The DVD provided the hazard start (when the hazard can be seen on the screen) and end times (when the driver must respond to the hazard) used for analysis. The area (not object size) that the pedestrians and vehicles occupied while considered a hazard was obtained by manually marking the rectangular bounding box formed from the hazard starting and ending frames of the video. Hazards covered an average area of $11^{\circ}$ (range: $3-25^{\circ}$ ) by $7^{\circ}$ (range: $2-17^{\circ}$ ). The median field-ofview and ring scotoma outer diameters of participant's non occluding bioptics were 8.8 and $41^{\circ}$, respectively.

Eleven of the 15 hazards were at least partially outside the binocular device scotoma of the partially occluding bioptic (as in Figure $1 b$ bottom). Four of the 15 were to the left or right (lateral) to the characters and blocked by the device scotoma (Figure $1 b$ top). Due to the unequal number of hazards and small number of lateral hazard trials, additional data from eight normally sighted subjects was collected. These subjects watched a total of 32 video clips using the partially blocking design: 16 with lateral hazards (expected to be blocked by the binocular device scotoma, Figure $1 b$ top) and 16 with vertical hazards (expected to be visible in the periphery above or below the device scotoma, Figure $1 b$ bottom). 


\section{Bioptic telescopes}

We tested two monocular telescope designs: a non-occluding telescope (such as the Ocutech VES mini (a 3.0× Keplerian) in Figure $2 a$ ) and a partially occluding telescope design (Ocutech VES K (a 4.0× Keplerian) in Figure 2c) which extends across both spectacle lenses blocking part of both eyes' view, thus creating a binocular device scotoma when looking through the telescope (Figure $2 d$ ). The purpose of testing the partially occluding telescope is to show that the fellow eye's compensation effect can be reduced if it is partially blocked.

Visually impaired participants used their own bioptic telescopes in the study (with a reading cap to focus at the testing distance if needed) and all except one used non-occluding monocular bioptics (Galilean or Keplerian). One participant owned a VES K telescope; therefore he used a VES mini for the 'non-occluding' condition and his VES K for the 'partially occluding' condition. With non-occluding bioptics, binocular perimetry found complete visual field except for two participants where overlap of the disease-caused scotoma in the fellow eye resulted in a binocular scotoma when overlapped with the ring scotoma, and similarly for all participants where the physiological blind spot overlapped with the telescope ring scotoma. Seven subjects used 3.0 Keplerian bioptics, two had a $4.0 \times$ Keplerian bioptic, one had a $2.2 \times$ Galilean bioptic, and one had a $6.0 \times$ Keplerian bioptic. One subject was a user of a centrally mounted (non bioptic) Galilean telescope and therefore used a VES mini bioptic for the 'non-occluding' condition.

\section{Participants}

Fifteen bioptic users with reduced central vision were recruited and 12 completed the study. Three failed to complete the study for nonvisual reasons. Inclusion criteria were visual acuity of $0.3 \log$ MAR (6/12 or 20/40 Snellen) to $1.0 \log$ MAR (6/60 or 20/200 Snellen) and prior use of a bioptic telescope. In addition, data were collected from eight normally sighted subjects in the same age range. Normally sighted subjects had visual acuity better than 0.2 $\log$ - MAR (6/9 or 20/30 Snellen).

The study was approved by the institutional review board at Massachusetts Eye and Ear and was conducted according to the tenets of the Declaration of Helsinki.

\section{Data analysis}

Hazard detections were recorded as correct if participants responded between the hazard start and end times, as defined in the UK training material. No grace period was provided at the end as none is provided in the UK training materials. For correct detections, detection rate (number of hazards identified/total number of hazards) and reaction time (period between hazard appearance and participant response) were determined. A point score based on the UK scoring method was calculated (https://www.gov.uk/driving-theory-test/how-thetheory-test-works). For this UK scoring each hazard time window was divided into five equal segments. The number of points scored depended on the participant response time, with earlier responses receiving higher scores. Therefore, a maximum of five points can be given for each hazard, and a maximum of 75 points for the total 15 hazards in each condition. High point scores can be achieved by high detection rates and short reaction 
times. The effects of the telescope, telescope design, and secondary reading task were analysed using Wilcoxon Signed Rank tests, as UK scores were not normally distributed (Shapiro-Wilk $=0.852, p=0.039$ ). To analyse the unequal number of hazards blocked by the binocular device scotoma and those partially outside, the ratio of points scored to the total points possible was used as a normalised value. Median detection rates and reaction times are reported in addition to the UK score. To evaluate whether hazard type affected performance, the ratio of UK points scored to total points possible for pedestrian and vehicle hazards were compared within conditions for visually impaired and normal vision subjects (there were more vehicle hazards than pedestrians). All comparisons were within subjects.

\section{Results}

\section{Sample characteristics}

Of the 12 bioptic users, five were current drivers, three former drivers, two with a learner's permit, and two non-drivers. Median time using the bioptic was 12.5 years [Interquartile range (IQR) 5.5-21 years]. There were no significant differences in age [47 (IQR 36-52) vs 47 (IQR 25-65) years, $p=0.91$ ] or gender (92\% vs 63\% male, $p=0.11$ ) between bioptic users and normally sighted controls, respectively. As expected, normally sighted controls had significantly higher contrast sensitivity [1.95 (IQR 1.8-2.0) vs 1.46 (IQR 1.3-1.78), $p=$ 0.008 ] and better visual acuity (telescope eye $20 / 17$ vs $20 / 100, p<0.001$; fellow eye $20 / 21$ vs $20 / 134, p<0.001$ ) than bioptic users.

\section{Hazard detection test performance}

Figure 3 summarises bioptic users' performance for UK point scores, detection rates, and reaction times for each of the four conditions. Figure 4 presents UK score data for the main effects.

\section{Effect of secondary reading task}

Performance measured by the UK score was significantly reduced with the addition of the secondary reading task, from 29 points with no reading task to 20 points with the reading task $(Z=-2.85, p=0.004 ;$ Figure $4 a)$. Detection rates were $83 \%$ and $67 \%$, respectively with false positive rates of $10 \%$ in both conditions.

\section{Effect of non-occluding bioptic}

UK scores were significantly reduced in the reading task with the non-occluding bioptic design from 20 points in the reading task with no bioptic to 16 points with the nonoccluding bioptic design $(Z=-2.59, p=0.01$; Figure $4 b)$. Detection rates were $67 \%$ and $50 \%$, respectively with false positive rates of $10 \%$ and $6.7 \%$.

\section{Effect of device scotoma}

Comparing bioptic users' performance for all hazards UK scores were significantly reduced with the partially occluding bioptic design, from 16 with the non-occluding bioptic to 9 with the partially occluding bioptic $(Z=-2.04, p=0.041$; Figure $4 c)$. Detection rates were $50 \%$ and $34 \%$, respectively with false positive rates of $6.7 \%$ in both conditions. 
While the overall performance with a partially occluding bioptic was reduced, it was necessary to further confirm whether the reduction was indeed due to the device scotoma. If confirmed, the finding can help prove the fellow eye's role in compensating. With a partially occluding bioptic, the hazard detection performance should be better for un-occluded areas than occluded areas. For the 12 subjects, there were only four hazards in occluded areas (lateral as in Figure $1 b$ top) and four in un-occluded areas (vertical as is Figure $1 b$ bottom). Because the small number of lateral/ vertical trials did not warrant analysis (one hazard corresponds to $25 \%$ detection rate), additional data was collected from eight normally sighted subjects wearing a partially occluding bioptic. Each subject was presented 16 vertical and 16 lateral hazards. Their detection rates and UK point scores were significantly higher for vertical compared to lateral hazards ( $53 \%$ vs $38 \%, Z=-2.13, p=0.035 ; 16$ vs 12 , $Z=-2.10, p=0.035$ ).

To ensure that the lateral hazards blocked by the device scotoma were not generally more difficult to detect than the others, visually impaired subjects' performance for lateral and other hazards in the reading task without bioptic and with the non-occluding bioptic conditions were compared. For either condition, there is no expectation of a difference in performance due to hazard location. Indeed, there was no significant difference in detection rate $(50 \%$ vs $59 \%, Z=-0.63, p=0.53)$ or normalised UK scores ( 0.19 vs $0.23, Z=-0.24$, p $=0.81$ ), suggesting that lateral hazards were not generally more difficult to detect than the others.

\section{Reading accuracy}

Reading accuracy was compared across instances when hazards were detected, present but not detected, and not present for the telescope conditions. If performance in the secondary reading task was impacted by hazard detection, we might expect reading accuracy to be lower when hazards were detected compared to not detected or not present. There was no significant difference in reading accuracy with the non-occluding bioptic when hazards were detected compared to not detected ( $68 \%$ vs $73 \%, Z=-1.276, p=0.202)$ or not present $(68 \%$ vs $70 \%, Z=-1.33, p=0.18$ ). Similarly, there was no significant difference with the partially occluding bioptic ( $68 \%$ vs $81 \%, Z=-0.80, p=0.42 ; 68 \%$ vs $73 \%, Z=-0.41, p=0.69$ ) respectively.

\section{Effect of test order}

The Freidman test was used to determine if there was an overall effect of the order of test administration revealing fatigue or learning effects. There was no significant effect of test order on UK scores for bioptic subjects $\left(\chi^{2}=2.24, p=0.53\right)$ or normally sighted controls $\left(\chi^{2}\right.$ $=2.25, p=0.52 ;$ Figure 5).

\section{Comparison to score required in UK for licensing}

The median UK point score for normally sighted subjects (averaged across all conditions) was 40 with only two of the eight subjects scoring over the 44 point passing mark used in the UK. Also, only one of the 12 bioptic participants scored above 44 points with no reading task and no bioptic; performance in the other three conditions was lower. UK point scores 
for bioptic users with no reading task were significantly lower than scores for normally sighted subjects ( 29 vs $40, U=20, p=0.031$ ).

\section{Type of hazard}

For bioptic users there was no significant difference in performance (normalised UK scores averaged across conditions) detecting pedestrian compared to vehicle hazards ( 0.25 vs 0.22 , $\mathrm{z}=-1.33, p=0.18$ ). Normally sighted subjects performed slightly better for pedestrian hazards compared with vehicle hazards ( 0.57 vs $0.52, \mathrm{z}=-1.96, p=0.05)$.

\section{Discussion}

The reduction in performance detecting hazards in the ring scotoma area with bioptics suggests that rivalry, particularly reduced predominance in the fellow eye, may occur in our more realistic experiment that uses videos of driving scenes. This effect was not found in previous perimetry-like testing procedures. ${ }^{15,16}$ Two possible predominance-reducing factors could account for the difference in findings. First, the hazard stimuli gradually develop, while the artificial stimuli in the perimetry-like procedure appeared abruptly. Sudden onset can capture predominance in rivalrous conditions, ${ }^{17}$ Secondly, the background of the current study was driving scenes in motion, while the background in previous studies was static. The conflicting motion between the telescope eye and fellow eye in the driving videos may have contributed to the increased predominance of the attended telescope view, as differences in motion velocity can affect binocular rivalry. ${ }^{22}$ A recent study ${ }^{23}$ involving hemianopic people wearing unilateral peripheral prism glasses, which created a rivalry condition similar to our current study, provides further support. Shen et al. ${ }^{23}$ found reduced target detection of the prism image when binocularly viewing driving videos (condition for rivalry) compared to monocular viewing (no rivalry). In contrast, detection rate differences were not found between binocular and monocular viewing on static images from the same videos in which spatial rivalry conditions existed but no conflicting motion was present.

Despite the effect of the ring scotoma on detection of realistic hazards, the current study did find that to some extent the fellow eye could compensate for the ring scotoma: 50\% detection rate with non-occluding telescope compared with $34 \%$ with the partially occluding telescope (as shown in Figure $3 b$ ). The additional data from normally sighted subjects provides further cause-and-effect evidence to confirm that, in the same fellow eye, the unoccluded visual field can detect hazards better than the occluded field (UK score 53\% vs $38 \%$ ). One reason why some lateral hazards were still detected might be that they were not completely blocked due to variable head tilting, which should be beneficial for users of partially-occluding telescopes. As the comparison is within subjects, the fact that data is from normally sighted subjects is irrelevant. Nevertheless, it has been shown that normally sighted subjects can quickly become proficient at using a bioptic. ${ }^{24}$

Binocular rivalry may play a contributing role in the compensation effect of the fellow eye. As perception alternates between the two eyes, predominance of the fellow eye enables fellow eye detection of hazards. It could also be possible that a voluntary attention shift between the two eyes allows subjects to detect some hazards with the fellow eye. However, our reading accuracy results do not support such an attention shift, as the reading accuracy 
when hazards were detected (68\%) was not significantly different from that when hazards were not present or not detected (70\% or $73 \%$ ). Participants seemed to focus more on the reading task as required.

Our study also revealed that the reading task alone reduced performance from 29 UK scoring points without reading to 20 points with the reading task (Figure $3 a$ ). It has been shown that a secondary task and increased attentional load reduces performance. ${ }^{25-27}$ The larger reduction in performance with the divided attention task alone (nine points on average) compared with the use of the telescope (four points) puts the effect of the ring scotoma in perspective. It appears to be only half as large as the effect of attention.

Even without the bioptic or divided attention task, it is interesting to note that performance for the majority of participants was below the UK passing mark, 44 points. Only one of the 12 bioptic users and two of the eight normally sighted subjects (averaged score across four video sets) reached this mark. The UK data for licensure candidates from 2011/2012 shows a pass rate of $85.7 \%$ (1 175986 of 1371500 drivers). UK drivers intensively prepare for the test (the training $\mathrm{CD}$ we used includes 400 videos), whereas our participants only received a short practice session. Therefore, the low scores of bioptic users and our normally sighted do not necessarily suggest they could not qualify for licensure in the UK, as hazard perception performance is known to improve with training. ${ }^{28}$ The failure of our normally sighted current drivers may cast doubt on the validity of the test but the subject numbers are too small for such between subject analyses. While the pass rate for participants in our study is lower than the UK pass rate, the use of this measure is still valid. The comparisons of interest are within subjects and UK scores account for both detection rate and reaction time providing a more complete measure of performance. Detection rates in our study are comparable with previous studies using similar hazard perception videos. ${ }^{29-31}$

The focus of this study was on evaluating detection in the area corresponding to the ring scotoma. In order to prevent subjects from perceiving cues of upcoming hazards from binocular viewing, the reading task started early, and lasted longer (about $5 \mathrm{~s}$ ) than in typical real world driving situations. When a hazard was missed in the experiment, it was missed during any portion of the long period of telescope viewing, which would be similar to actual bioptic use behaviours. In actual driving, bioptic users typically make short glances (approximately $1 \mathrm{~s}$ ) through the telescope, ${ }^{32}$ and they may encounter slowly developing and suddenly appearing hazards. Presumably, for gradually developing hazards, such as a visible, crossing cyclist at a large eccentricity, bioptic users can have a few chances to spot the potential hazards in time after bioptic use, even if it is missed during the brief telescope viewing. For suddenly appearing and imminent hazards, such as a pedestrian emerging behind an obstructing truck, the detection may be impacted, as shown in this study. Since the ring scotoma is present only during infrequent bioptic use events, it is possible that the overall impact of the ring scotoma in the real world is smaller, relative to other human factors, such as experience. A recent study suggested that visual acuity and contrast sensitivity are not predictors of bioptic driving performance. ${ }^{33} \mathrm{We}$ are conducting naturalistic bioptic driving studies to better understand how the use of bioptic telescopes impacts safety in daily driving. ${ }^{32}$ 


\section{Conclusion}

We found that the use of the telescope did reduce but did not completely prevent the detection of realistic hazards that fall in the ring scotoma even though they were visible to the fellow eye while subjects viewed through the telescope. Our results suggest that divided attention due to a secondary reading task may play a larger role in reducing hazard detection performance than the effect of the ring scotoma, yet these effects are cumulative.

\section{Acknowledgements}

Akarsha Ramani and Dekuang Yu wrote MATLAB scripts and Jih-Ping Chern developed the data collection software. Supported in part by grants: R21AG034553-S1 (GL), R01AG041974 (GL), R01EY12890 (EP) and P30EY003790.

\section{References}

1. Feinbloom W. Driving with bioptic telescopic spectacles. Am J Optom Physiol Opt. 1977; 54:3542. [PubMed: 860749]

2. Korb DR. Preparing the visually handicapped person for motor vehicle operation. Am J Optom Arch Am Acad Optom. 1970; 47:619-628. [PubMed: 5273102]

3. Clarke N. An evaluation of the traffic safety risk of bioptic telescopic lens drivers: Research and Development Branch Division of Program and Policy Administration California Department of Motor Vehicles. 1996

4. Lippmann O, Corn AL, Lewis MC. Bioptic telescopic spectacles and driving performance: a study in Texas. J Vis Impair Blind. 1988; 82:182-187.

5. Owsley C. Driving with bioptic telescopes: organizing a research agenda. Optom Vis Sci. 2012; 89:1249-1256. [PubMed: 22863791]

6. Melis-Dankers B, Kooijman A, Brouwer W, et al. A demon-stration project on driving with reduced visual acuity and a bioptic telescope system in the Netherlands. Vis Impair Res. 2008; 10:7-22.

7. Kooijman AC, Melis-Dankers BJM, Peli E, et al. The introduction of bioptic driving in the Netherlands. Vis Imp Res. 2008; 10:1-6.

8. Bowers AR, Apfelbaum DH, Peli E. Bioptic telescopes meet the needs of drivers with moderate visual acuity loss. Invest Ophthalmol Vis Sci. 2005; 46:66-74. [PubMed: 15623756]

9. Fonda G. Bioptic telescopic spectacle is a hazard for operating a motor vehicle. Arch Ophthalmol. 1983; 101:1907-1908. [PubMed: 6651597]

10. Keeney AH. Field loss vs central magnification. Arch Ophthalmol. 1974; 92:273. [PubMed: 4412945]

11. Kelleher DK, Mehr EB, Hirsch MJ. Motor vehicle operation by a patient with low vision: a case report. Am J Optom Arch Am Acad Optom. 1971; 48:773-776. [PubMed: 5285989]

12. Fetchenheuer I, Peli E, Woods RL. Functional visual fields of monocular bioptic telescopes (abstract). The 7th International Conference on Low Vision: Activity and Participation. 2002:81.

13. Jose R, Ousley BA. The visually handicapped, driving with bioptics - some new facts. Rehabil Optom. 1984; 2:2-5.

14. Blake R. A primer on binocular rivalry, including current controversies. Brain and Mind. 2001; 2:5-38.

15. Doherty AL, Bowers AR, Luo G, Peli E. Object detection in the ring scotoma of a monocular bioptic telescope. Arch Ophthalmol. 2011; 129:611-617. [PubMed: 21555615]

16. Doherty AL, Bowers AR, Luo G, Peli E. The effect of strabismus on object detection in the ring scotoma of a monocular bioptic telescope. Ophthalmic Physiol Opt. 2013; 33:550-560. [PubMed: 23639041]

17. Blake R, Logothetis N. Visual competition. Nat Rev Neurosci. 2002; 3:13-21. [PubMed: 11823801] 
18. Horswill, MS.; McKenna, FP. Drivers' hazard perception ability: situation awareness on the road. In: Banbury, S.; Tremblay, S., editors. A cognitive approach to situation awareness: theory and application. Ashgate Publishing; Aldershot: 2004. p. 155-175.

19. Sagberg F, Bjornskau T. Hazard perception and driving experience among novice drivers. Accid Anal Prev. 2006; 38:407-414. [PubMed: 16313881]

20. Shahar A, Alberti CF, Clarke D, Crundall D. Hazard perception as a function of target location and the field of view. Accid Anal Prev. 2010; 42:1577-1584. [PubMed: 20728606]

21. Vargas-Martin F, Garcia-Perez MA. Visual fields at the wheel. Optom Vis Sci. 2005; 82:675-681. [PubMed: 16127332]

22. Blake R, Zimba L, Williams D. Visual motion, binocular correspondence and binocular rivalry. Biol Cybern. 1985; 52:391-397. [PubMed: 4052502]

23. Shen J, Peli E, Bowers A. Peripheral prism glasses: effects of moving and stationary backgrounds. Optom Vis Sci. 2015; 92:412-420. [PubMed: 25785533]

24. Tadin D, Lappin JS, Sonsino J. Recognition speed using a bioptic telescope. Optom Vis Sci. 2008; 85:1135-1141. [PubMed: 19050468]

25. Chaparro A, Wood JM, Carberry T. Effects of age and auditory and visual dual tasks on closedroad driving performance. Optom Vis Sci. 2005; 82:747-754. [PubMed: 16127341]

26. Strayer DL, Drews FA. Cell-phone-induced driver distraction. Curr Dir Psychol Sci. 2007; 16:128131.

27. Strayer DL, Johnston WA. Driven to distraction: dual-Task studies of simulated driving and conversing on a cellular telephone. Psychol Sci. 2001; 12:462-466. [PubMed: 11760132]

28. McKenna FP, Crick J. Hazard perception in drivers: a methodology for testing and training Crowthorne: Transport Research laboratory1994 Contract No. Contractor Report. :313.

29. Preece MH, Horswill MS, Geffen GM. Assessment of drivers' ability to anticipate traffic hazards after traumatic brain injury. J Neurol Neurosurg Psychiatry. 2011; 82:447-451. [PubMed: 20884679]

30. Rauscher FG, Chisholm CM, Crabb DP, et al. Central Scotomata and Driving. Road Safety Research Report. Department of Transport: London, 2007 Dec. Report No.: 79.

31. Wetton MA, Horswill MS, Hatherly C, Wood JM, Pachana NA, Anstey KJ. The development and validation of two complementary measures of drivers' hazard perception ability. Accid Anal Prev. 2010; 42:1232-1239. [PubMed: 20441837]

32. Luo G, Peli E. Recording and automated analysis of naturalistic bioptic driving. Ophthalmic Physiol Opt. 2011; 31:318-325. [PubMed: 21410498]

33. Dougherty BE, Flom RE, Bullimore MA, Raasch TW. Vision, training hours, and road testing results in bioptic drivers. Optom Vis Sci. 2015; 92:395-403. [PubMed: 25946098] 
(a)

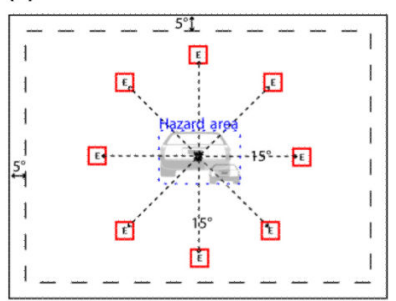

(b)

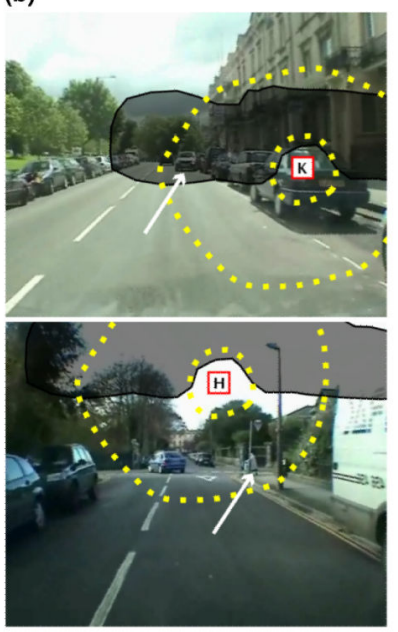

(c)

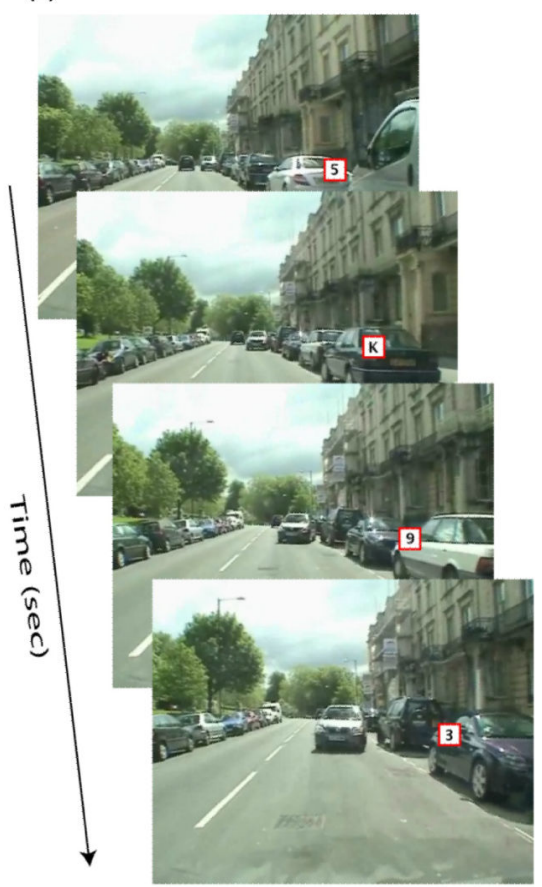

Figure 1.

(a) An illustration of character placement relative to the hazard (car). Characters are randomly assigned to one of eight directions around the area occupied by the hazard, $15^{\circ}$ from the centre of the hazard area and at least $5^{\circ}$ from the edge of the screen. (b) Character sequences were positioned $15^{\circ}$ from the hazard centre such that when sighted through the telescope, the hazard falls in the monocular ring scotoma area (between the dashed yellow circles). The grey shaded areas mark the binocular scotoma created by the partially occluding VES K device design. The top example shows a lateral hazard (white car) which falls in the binocular scotoma area of the VES K design but only in the monocular scotoma of the non-occluding design. The bottom example shows a vertical hazard (pedestrian) that falls outside the binocular device scotoma. (c) One or two letters appeared amid a sequence of numbers changing every $320 \mathrm{~ms}$ for the duration of the hazard. 
(a)

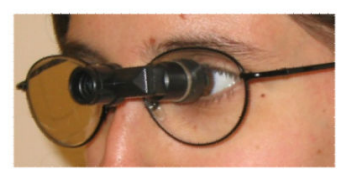

(b)

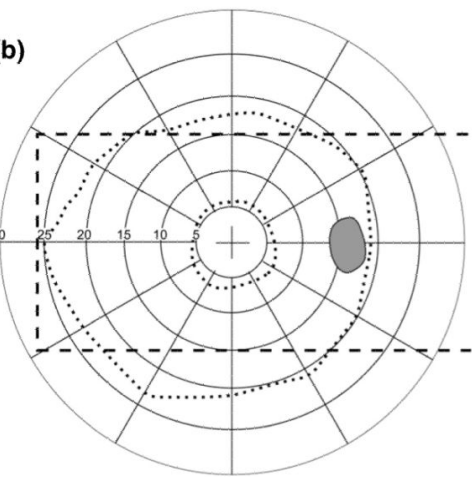

(c)

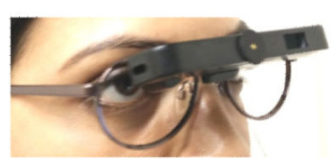

(d)

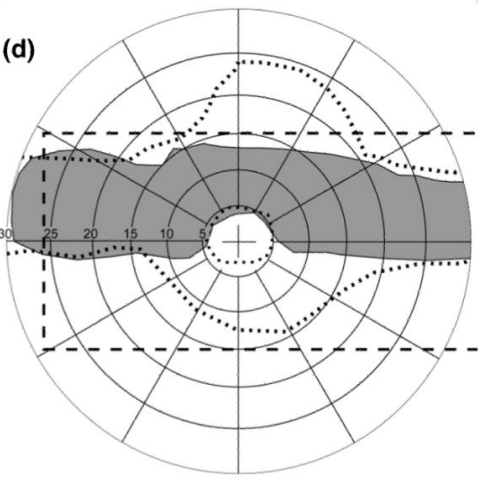

Figure 2.

(a) A person viewing through a 3.0 $\times$ VES mini bioptic fitted on the left eye; (b) Binocular visual field of a bioptic user viewing through the $3 \times$ VES mini left bioptic telescope. The only binocular scotoma is the overlap of the ring scotoma with the physiological blind spot of the fellow eye. The area bound between the two dotted circular regions represents the monocularly measured ring scotoma. (c) A person viewing through a $4 \times$ VES K right bioptic telescope. (d) Binocular visual field while viewing through the $4 \times$ VES K bioptic. The telescope housing creates a binocular scotoma (shaded grey). The area bound between the two dotted circles represents the monocularly measured ring scotoma and the monocular telescope housing scotoma. The dashed line in (b) and (d) represents the view through a standard car windshield. ${ }^{21}$ 

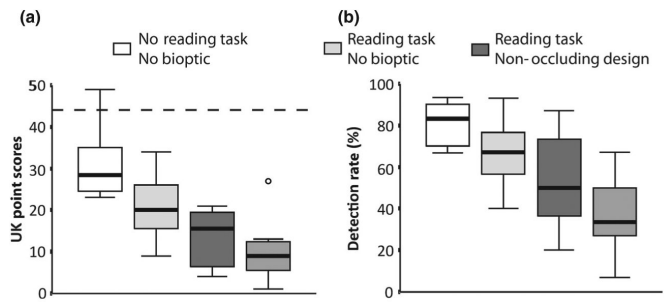

(c)

$\square$ Reading task

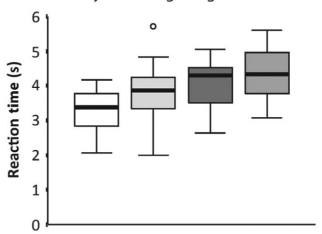

Figure 3.

Bioptic users' performance in each of the four conditions. (a) UK point scores, dashed line indicating UK passing mark (b) detection rates (c) reaction times. Thick horizontal line within the box is the median; the vertical extent of the box is the interquartile range (IQR); whiskers represent the data range with outlier excluded; and circles are outliers (1.5-3 IQR). 

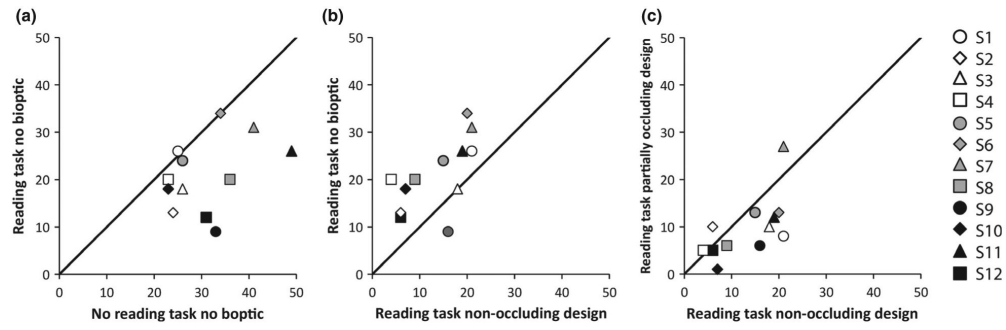

Figure 4.

UK point scores for the main effects. (a) UK scores were significantly lower with the addition of the reading task. (b) UK scores were significantly lower with the non-occluding bioptic design compared to the reading task with no bioptic. (c) UK scores were significantly lower with the partially occluding design compared to the non-occluding bioptic design. Note that higher subject number codes for poorer visual acuity, which is also coded by darkness of the symbols. 


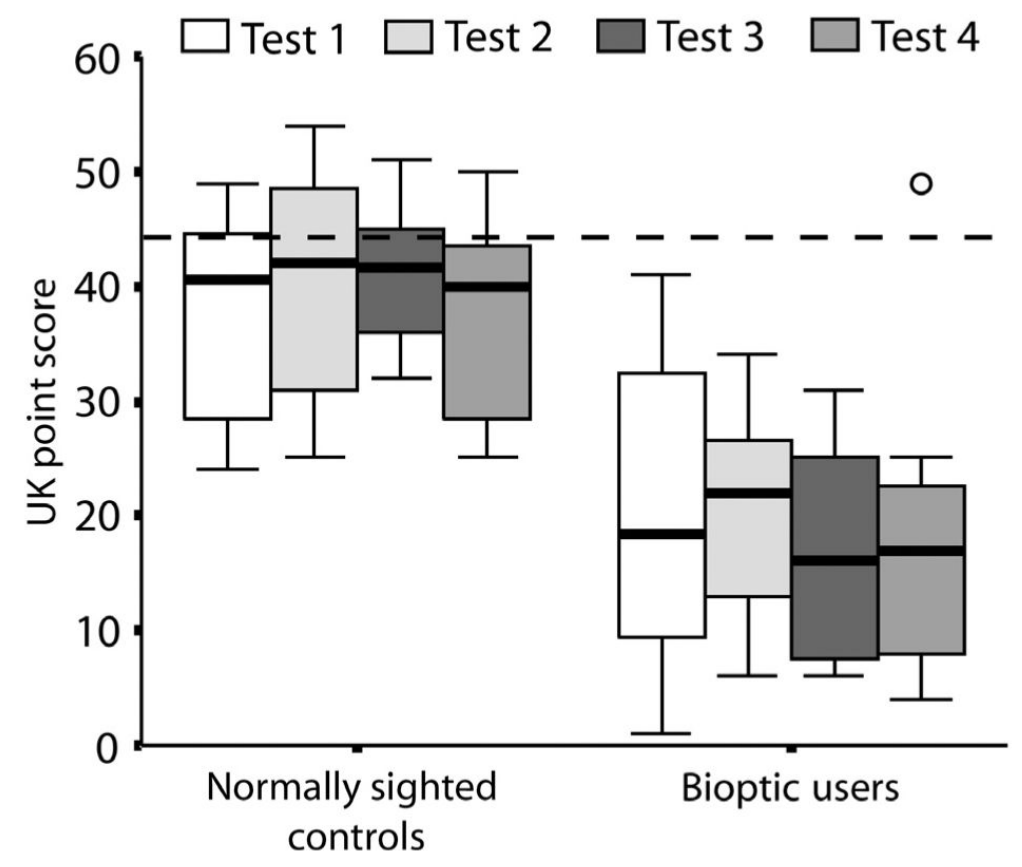

Figure 5.

UK scores were not significantly different across order tests were administered, suggesting no effects of learning or fatigue for bioptic users or normally sighted controls. The notations in this graph are the same as in Figure 3. 
Table 1

Visual acuity (in imperial Snellen) and character sizes used for each participant

\begin{tabular}{llllllll}
\hline Participant & $\begin{array}{l}\text { Telescope eye } \\
\text { VA (without } \\
\text { telescope) }\end{array}$ & $\begin{array}{l}\text { VA with } \\
\text { non-occluding } \\
\text { bioptic }\end{array}$ & $\begin{array}{l}\text { Non-occluding } \\
\text { bioptic } \\
\text { magnification }\end{array}$ & $\begin{array}{l}\text { VA with } \\
\text { 4× partially } \\
\text { occluding bioptic }\end{array}$ & $\begin{array}{l}\text { Letter size } \\
\text { without } \\
\text { bioptic }\end{array}$ & $\begin{array}{l}\text { Letter size } \\
\text { with both } \\
\text { bioptics }\end{array}$ & $\begin{array}{l}\text { Driving } \\
\text { status }\end{array}$ \\
\hline 1 & $20 / 63$ & $20 / 26$ & 3.09 & $20 / 18$ & $1.2^{\circ}$ & $0.5^{\circ}$ & Non driver \\
2 & $20 / 63$ & $20 / 27$ & 3.09 & $20 / 25$ & $1.2^{\circ}$ & $0.5^{\circ}$ & Current \\
3 & $20 / 76$ & $20 / 26$ & 3.09 & $20 / 22$ & $1.2^{\circ}$ & $0.5^{\circ}$ & Current \\
4 & $20 / 80$ & $20 / 22$ & 4.09 & $20 / 18$ & $1.2^{\circ}$ & $0.5^{\circ}$ & Permit \\
5 & $20 / 84$ & $20 / 38$ & 3.09 & $20 / 31$ & $1.8^{\circ}$ & $0.8^{\circ}$ & Former \\
6 & $20 / 95$ & $20 / 31$ & 3.09 & $20 / 18$ & $1.2^{\circ}$ & $0.5^{\circ}$ & Permit \\
7 & $20 / 105$ & $20 / 26$ & 4.09 & $20 / 24$ & $1.2^{\circ}$ & $0.5^{\circ}$ & Current \\
$8^{a}$ & $20 / 110$ & $20 / 38$ & 3.09 & $20 / 25$ & $1.4^{\circ}$ & $0.6^{\circ}$ & Non driver \\
9 & $20 / 145$ & $20 / 57$ & 2.29 & $20 / 35$ & $1.4^{\circ}$ & $0.8^{\circ}$ & Current \\
$10^{b}$ & $20 / 152$ & $20 / 50$ & 3.09 & $20 / 24$ & $1.9^{\circ}$ & $0.8^{\circ}$ & Former \\
11 & $20 / 166$ & $20 / 52$ & 3.09 & $20 / 38$ & $1.4^{\circ}$ & $0.6^{\circ}$ & Former \\
$12^{c}$ & $20 / 230$ & $20 / 36$ & 6.09 & $20 / 66$ & $1.9^{\circ}$ & $0.8^{\circ}$ & Current \\
\hline
\end{tabular}

${ }^{a}$ Own bioptic is centrally mounted. Used 3.09 mini for non-occluding telescope condition.

${ }^{b}$ VES K partially occluding bioptic user.

${ }^{c}$ Subject's binocular unaided visual acuity meets the 20/200 inclusion criterion. 